\title{
LA LIBERTAD DE LOS DOMINADOS. APORTES Y LÍMITES DE LA TEORÍA DEL PODER DE JOHN SEARLE
}

\author{
Pablo Beytía \\ Centro de Investigación Social de TECHO-Chile
}

\begin{abstract}
RESUMEN: Las teorías sobre el poder presentan un dilema teórico. Algunas consideran que mientras mayor es el grado de poder de un agente, menor libertad tienen quienes quedan sometidos a él. Otras sugieren justamente lo contrario: la mayor libertad de los dominados implica un superior poder del agente dominante. Este artículo revisa la teoría del poder de John Searle, identificando los elementos que podrían aportar en esta discusión. Su propuesta no acepta la eliminación de la voluntad en los agentes sometidos, pero sí considera en ellos una reducción de su libertad "negativa".

Palabras clave: Poder, libertad, dominación, coacción, John Searle. RECIBIDO: enero 2014; ACEPTADO: junio 2014.

\section{FREEDOM OF THE DOMINATED. CONTRIBUTIONS AND LIMITS FROM THE POWER THEORY OF JOHN SEARLE}

ABSTRACT: Theories about power present a theoretical dilemma. Some believe that the greater an agent's degree of power is, less will be the freedom of those subject to that agent. Others suggest just the opposite: greater freedom of the dominated implies a greater

Pablo Beytía es sociólogo y magíster en sociología, Pontificia Universidad Católica de Chile. Profesor Pontificia Universidad Católica de Chile. Director del Centro de Investigación Social de TECHO-Chile. Profesor Universidad Alberto Hurtado. Candidato a magíster en filosofía, Universidad de Chile. Email: ptbeytia@uc.cl. 
power of the dominant agent. This article reviews John Searle's theory of power and identifies elements that could contribute to this discussion. Searle's theory does not accept the elimination of will in the subjugated agents, but it does consider that they have a reduced 'negative' liberty. Searle does not ask whether said agents' freedom might possibly increase, which explains the theoretical invisibility of some relevant processes in power.

KEYwords: Power, freedom, domination, coaction, John Searle.

RECEIVED: January 2014; ACCEPTED: June 2014.

\section{INTRODUCCIÓN}

uego de haber realizado penetrantes estudios sobre filosofía del lenguaje y de la mente, el filósofo estadounidense John Searle comenzó a interesarse enérgicamente por "ciertos rasgos estructurales generales de la cultura humana" $(1997,23)$. El cuestionamiento por la construcción de la realidad y el mundo social se transformó - como él mismo afirmó - en cierta "obsesión” personal, que determinó la elaboración de dos grandes frutos: su libro de 1997, La construcción de la realidad social, y su contribución más amplia a la teoría social, realizada en Making the Social World: The Structure of Human Civilization (2010).

En este último escrito, Searle se introdujo en un tema poco común para la filosofía angloparlante, aunque de extenso trato en la tradición europea continental: el poder. Este interés no podría ser calificado como casual, al menos considerando el alto impacto que han tenido en la teoría social los estudios clásicos de Marx, Weber, la Escuela de Frankfurt y Foucault, más el acercamiento decisivo a esta temática de algunos académicos anglosajones contemporáneos, como Michael Mann (1986 y 1993) y Anthony Giddens (2006 y 2007). La importancia de este concepto en la teoría social ha llegado a ser tal, que Manuel Castells inició su elaborado estudio sobre Comunicación y poder simplemente afirmando que "el poder es el proceso fundamental de la sociedad" $(2009,33)$, ya que desde aquél se precisan los valores e instituciones que definen a ésta.

Debido a las miles de páginas que podrían reunirse sobre este tema, es natural que se identifiquen algunos puntos de discusión entre los distintos autores. Por ejemplo, el poder se ha entendido como un conjunto de medios (Hobbes 1996), una probabilidad (Weber 2008), 
una capacidad (Mann 1986; Giddens 2007; Castells 2009) o un medio generalizado de comunicación (Parsons 1999; Luhmann 2005). Por otra parte, algunos autores enfatizan la subjetividad o voluntad del agente de poder (Weber 2008; Castells 2009), mientras otros destacan la constitución del fenómeno como una magnitud objetiva y externa a los individuos (Foucault 2009; Schmitt 2010); notando esta tensión, hay quienes postulan que el poder, considerado desde el horizonte de acción en una vida humana, es previo incluso a la distinción individual entre sujeto y objeto (Giddens 2007).

Dentro de las discusiones teóricas más interesantes, este ensayo abordará una de gran relevancia: la problemática relación entre poder y libertad de aquellos que se someten a él. Por un lado, algunos autores sostienen que el grado de poder de un agente A se vincula negativamente con la libertad de un agente $B$, quien está sujeto al poder de $A$. Así, por ejemplo, Castells $(2009,37)$ especifica:

El empoderamiento de los actores sociales no puede separarse de su empoderamiento contra otros actores sociales [...]. El poder para hacer algo [...] es siempre el poder de hacer algo contra alguien, o contra valores e intereses de ese "alguien" que están consagrados en los aparatos que dirigen y organizan la vida social.

Esta versión ha sido fundamentada en definiciones del poder que enfatizan la capacidad de influencia de los empoderados, como la que célebremente estableció Robert Dahl en 1957. Según el politólogo estadounidense, un agente A tiene poder sobre B, en la medida en que A puede conseguir que $\mathrm{B}$ haga algo que no haría de otra manera (Dahl 1957, 201-203). Como ha especificado Rivas (2013), esta definición supone una relación negativa entre poder y libertad de los dominados por él: el poder de A implicaría la no libertad de B, y la libertad de B involucraría el no poder de A. Esta lógica ha llevado a algunos autores, como Zygmunt Bauman, a desdibujar la separación conceptual entre poder y libertad, proponiendo que "la división del poder [...] no consiste en nada más que la distinción entre una elección irrestricta y una elección reducida al mínimo existencial; la distinción entre libertad y no libertad" (2007, 57).

A esta comprensión de la relación entre poder y libertad de quienes se someten a él puede contraponerse un punto de vista que destaca los 
procesos de comunicación entre los agentes. Esta perspectiva enfatiza que el poder es un fenómeno relacional, que "surge de la capacidad que tienen los hombres no solamente para actuar o hacer cosas, sino también para concertarse con los demás y actuar de acuerdo con ello" (Arendt 1970, 41). Aquí la relación entre poder y libertad de los dominados es contrapuesta a la señalada anteriormente: el grado de poder de un agente A se vincula positivamente con la libertad de B, quien está sujeto al poder de $A$. Luhmann $(2005,14)$ ha sido probablemente quien mejor ha aclarado esta propuesta:

El poder también supone apertura a otras acciones posibles por parte del ego afectado por el poder. [...] El poder es mayor si es capaz de mantenerse incluso a pesar de alternativas atractivas para la acción o inacción. Y sólo puede aumentarse junto con un aumento de la libertad por parte de cualquiera que esté sujeto al poder.

Considerando este debate sobre la relación entre el grado de poder de un agente y la libertad de aquellos que se someten a él, en este ensayo mostraré los aportes y tomas de posición que pueden ser deducidos de la teoría del poder de John Searle. Si bien este autor dedica muchas páginas a detallar el funcionamiento de fenómenos como la intencionalidad o la coacción, lo cierto es que no desarrolla explícitamente el vínculo entre el poder de un agente y la libertad de aquellos que se someten a él. Tampoco los comentadores de su obra han elaborado literatura que interprete en profundidad este asunto. No obstante, el grado de libertad de los dominados ha sido un tema vital en la teoría política moderna, al menos desde que Hobbes enlazó positivamente el poder con la libertad y la dominación de otros individuos, ${ }^{1}$ dedicando una sección de su Leviatán a indagar en la libertad efectiva de los súbditos. Hoy esta problemática ha tomado una renovada importancia, especialmente debido a quienes, como Michael Walzer (2004) o Philip Pettit (1997), pro-

${ }^{1}$ Hobbes (1996, ca XIV) entendió la libertad como "ausencia de impedimentos externos, impedimentos que generalmente reducen parte del poder que un hombre tiene de hacer lo que quiere", vinculando directamente la reducción de poder con la reducción de libertad. Por otra parte, que haya entendido el poder de un hombre como "sus medios presentes para obtener algún bien manifiesto futuro", lo llevó a proponer que "tener siervos es poder", lo que enlaza el poder con la dominación (Hobbes 1996, ca X). 
mueven que la política realice el ideal de una sociedad libre, entendida fundamentalmente como un espacio ausente de dominación.

En lo que sigue, intentaré demostrar que la teoría del poder de Searle presume la mantención de la voluntad en los agentes dominados, pero no se pregunta por el grado variable de alternativas de acción en dichos agentes, lo cual acarrea la invisibilidad teórica de algunos procesos de poder ${ }^{2}$. Además, esta teoría exhibe dificultades para representar a una u otra postura en esta discusión, a pesar de que, si se considera la noción de "libertad negativa" planteada por Erich Fromm e Isaiah Berlin, estaría más cercana a quienes defienden que el poder disminuye la libertad de los dominados.

En una primera sección abordaré la teoría del poder de John Searle, identificando las consecuencias que tiene para entender el grado de libertad de los agentes sometidos al poder y el grado de poder que poseen los agentes dominantes. Posteriormente, analizaré el papel de la coacción en esta teoría, especificando en qué aporta para la comprensión de la libertad de quienes están sometidos al poder. Finalmente, observaré globalmente la visión de Searle sobre la libertad de los agentes dominados, fundamentalmente a partir de la distinción entre libertad positiva y negativa.

\section{CAPACIDAD DE ACTUAR, INTENCIONALIDAD Y MANERAS DE EJERCER EL PODER}

Si bien Searle reconoce la existencia de poder al margen de los procesos humanos, decide concentrar sus esfuerzos teóricos en las relaciones producidas entre personas e instituciones. Para él, la noción de poder - entendida en el más amplio sentido - es equivalente a la de capacidad o habilidad; ello implica que podría existir poder en una forma potencial y latente, es decir, sin ser ejercido por el agente que lo posee $^{3}$ (Searle 2010, 145).

${ }^{2}$ Específicamente dos procesos: a) las prácticas de poder que se realizan abriendo y no cerrando posibilidades de acción, y b) los cambios en el grado de poder producidos por variaciones en la cantidad de posibilidades de los agentes dominados.

${ }^{3}$ Otra forma de latencia o imperceptibilidad del poder, admitida por Searle, sucede cuando los agentes sometidos a él desean actuar de la misma manera que les impone el agente empoderado. 
El concepto que él describe -al igual que el delineado por Giddens (2007) — estaría asociado lógicamente a la noción de acción, ya que, según Searle $(2010,146)$, sería eficazmente descrito por dos tipos de sintaxis: a) el agente A tiene poder — capacidad o habilidad— de hacer una acción X; b) el agente A tiene poder sobre otro agente B con respecto a una acción X. Como puede observarse, en ambas formulaciones la acción sería aquello hacia lo que el poder se dirige, y sólo en la segunda sintaxis se caracteriza directamente el poder entre personas, es decir, una relación de dominación ${ }^{4}$.

Este primer acercamiento es sin duda amplio. Si sólo nos quedáramos con esta definición, toda acción involucraría poder, ya que cada actuar supone una capacidad para dicho actuar. En otras palabras, el poder sería equivalente a la "capacidad transformadora de la acción", que es justamente lo que propone inicialmente Giddens (2007, 136-137). No obstante, Searle no tarda en sugerir que no todo actuar implica un ejercicio de poder, ya que, para él, es indispensable que dicho ejercicio se vincule a una intención de conseguir algo (Searle 2010, 148). A pesar de que el filósofo reconoce que normalmente el ejercicio del poder desarrolla consecuencias no intencionales, sostiene enfáticamente que no puede sostenerse la presencia de poder ejercido a menos que exista una intención asociada a una determinada acción.

Cuando Searle menciona la intencionalidad, hay que aclarar que no se refiere a un proceso necesariamente consciente. Específicamente, se trata de "la capacidad de la mente para representar objetos y estados de cosas mundanos distintos a uno mismo" (Searle 1997, 26), una habilidad que no implica una pretensión en el actuar, pero sí que las representaciones estén dirigidas hacia algo. De tal modo, las creencias y los deseos serían intencionales — están dirigidos hacia algo—, y también

${ }^{4}$ El entendimiento de la dominación como un fenómeno de poder acotado a personas dadas - y no como fenómeno abstracto- proviene de Max Weber (2008). No obstante, éste último asume dicho concepto como una "probabilidad de encontrar obediencia a un mandato determinado", y este mandato no es necesario para que exista poder según la teoría de Searle. Por ello, cuando en este artículo se mencione el concepto "dominación" o "agente dominado", únicamente se estará haciendo referencia a la aplicación del poder entre personas específicas, sin asumir del todo la perspectiva weberiana. Tampoco se asumirá la propuesta de Foucault (1999), que implica entender los hechos de dominación como relaciones de poder que "en lugar de ser móviles y permitir a los diferentes miembros una estrategia que los modifique, se encuentran bloqueadas y fijadas". 
podría existir intencionalidad inconsciente (como la creencia en que una persona tiene un cargo público, a pesar de que no estemos pensando en ello en este mismo instante).

Añadiendo la intencionalidad a las sintaxis previamente señaladas, podríamos resumir que para Searle el ejercicio de poder entre personas o instituciones tiene la siguiente estructura: $A$ tiene poder sobre $B$ con respecto a la acción $X$, si y sólo si A puede intencionalmente conseguir que B haga lo que A quiere con respecto a la acción X (Searle 2010, 151). Partiendo de esta fórmula general, el filósofo estadounidense distingue dos maneras lógicas en que se puede desplegar el poder entre personas o instituciones que, como se verá posteriormente, nos llevan directamente a la discusión sobre la libertad de los agentes dominados.

Una primera manera de ejercitar el poder entre personas e instituciones es producida, según Searle $(2010,151)$, cuando el agente empoderado consigue que el sometido quiera hacer algo que podría no haber querido hacer de otra manera. Se trata, entonces, de ejercer intencionalmente una influencia en la voluntad, ${ }^{5}$ lo cual contrasta con la clásica definición otorgada por Max Weber (2008, 23): “Poder significa la probabilidad de imponer la propia voluntad, dentro de una relación social, aun contra toda resistencia y cualquiera que sea el fundamento de esa probabilidad". Como puede observarse, ambas definiciones son similares en cuanto a la estructura de la relación de poder y los resultados que suponen, aunque tienen una diferencia esencial: mientras Weber enfatiza que el agente empoderado es quien posee la voluntad e impone el curso de la acción, Searle considera que el agente sometido quiere hacer lo que el agente poderoso intenta, lo cual supone un ejercicio de voluntad.

Al comparar ambas definiciones de poder se esconde entonces un problema profundo: ¿puede decirse, siguiendo la terminología de Searle, que el agente dominado ha reducido su libertad? A mi parecer, ello no es posible, si consideramos únicamente esta manera de ejercer la dominación. "Conseguir querer" equivaldría a influir intencionalmente en la voluntad, y en esa influencia no se limitan las posibilidades de acción del agente dominado, sino que se intenta que él mismo proceda con una decisión definida por el poderoso. Aquí el concepto de poder estaría

${ }^{5}$ Searle $(2010,148)$ considera que no toda influencia es un ejercicio de poder, sino sólo aquella que es efectuada de manera intencional. 
más cercano al de acciones como "incitar" o "favorecer" que al "imponer" de Max Weber o al "obedecer" que destaca Carl Schmitt (2010)

Cabe destacar que Searle, cuando desarrolla esta manera de ejercer el poder, no tematiza los diferentes grados de libertad que podrían tener los agentes dominados, ni la influencia que ello tendría en el nivel de poder de quien "intenciona" la acción. En este caso, el grado de libertad del dominado se asume como constante: no podemos decir que disminuye — después de todo ambos agentes mantienen su voluntad-, pero tampoco se analiza la posibilidad de un aumento, lo cual tendría efectos (que posteriormente analizaremos) en el grado de poder del agente que lo posee.

La segunda manera de ejercer poder entre personas e instituciones se aplica, según Searle, cuando el agente empoderado presenta a otros agentes las opciones que quiere conseguir como únicas disponibles; en otras palabras, quien es sometido por el poder quiere hacer una acción porque un agente ha impedido que él vea otras posibilidades (Searle 2010, 151). Se trata, principalmente, de un ocultamiento de alternativas, que recuerda la definición elaborada por Niklas Luhmann (2005) del poder como medio de comunicación. Para el sociólogo alemán, la función de todo medio de comunicación es transmitir complejidad reducida, cosa que también sucedería en el caso del poder, considerado como "algo que limita la gama de selecciones del otro" (Luhmann 2005, 17).

Ambos autores destacan que el proceso de seleccionar qué alternativas observan los agentes es ya un ejercicio de poder - a pesar de que no se esté imponiendo una opción en específico-. Esto es muy claro, por ejemplo, al analizar la influencia de los medios masivos de comunicación: ellos no determinan directamente lo que el público piensa, aunque sí podrían influenciar sobre qué lo hace - estableciendo un universo de temas para la discusión-. En ese sentido, el grado de poder de la prensa y los canales de televisión - por nombrar algunos medios masivos - se jugaría más en la selección y ocultamiento de temas, que en la imposición de ideologías o pensamientos específicos.

A pesar de la similitud entre los procesos de poder destacados por ambos autores, Luhmann observa un asunto muy interesante en el cual

${ }^{6}$ Declara Schmitt $(2010,21)$ en su Diálogo sobre el poder y el acceso al poderoso: "Es sólo porque hay hombres que obedecen a otro hombre que a éste le es concedido el poder. Si dejan de obedecerle, el poder desaparece por sí solo". 
no repara Searle. Para el sociólogo alemán, es necesario distinguir los grados de poder según la diversidad de alternativas que poseen los agentes vinculados. "El poder aumenta con la libertad en ambas partes" - diagnostica Luhmann $(2005,15)$-, dado que el poder de quien lo posee es mayor si le permite tomar decisiones más diversas, pero también "si puede hacer esto con un compañero que, por su parte, posee varias alternativas diferentes". En otras palabras, Luhmann sugiere que mientras más libres son los agentes dominados, mayor poder está ejerciendo quien lo posee. Esto es vital, ya que efectivamente se necesitan menores recursos para guiar intencionalmente la acción de alguien que tiene dos o tres alternativas, a la de alguien con una inmensa gama de posibilidades; no es equivalente la capacidad de ocultar una opción, a la de ocultar cien. Si bien Searle da un paso teórico en esta dirección al defender que "todo poder viene desde abajo" — es decir, desde quienes se someten a él一, a pesar de que se ejerza "desde arriba" (Faigenbaum 2003, 127), lo cierto es que no llega a preguntarse por el efecto que tendría en el grado de poder el cambio en el grado de libertad de quienes se someten a él.

El mérito de Luhmann es haber resaltado la importancia de distinguir grados de libertad entre los agentes dominados, lo cual permite observar otro proceso de poder que queda oculto en la terminología de Searle. En el proceso de manejo de alternativas, no sólo se puede ejercer poder ocultando opciones - como destaca el filósofo estadounidense-, sino que también desocultándolas. Imaginemos, por ejemplo, que un agente B está encarcelado por intentar matar al presidente de un país; años después, existe un poderoso agente $\mathrm{A}$, que también quiere al presidente muerto y, para lograr su cometido, logra que el agente B salga de la cárcel; ello tiene como consecuencia el asesinato del presidente por parte del agente B. En esta situación, el agente de poder (A) también está manejando intencionalmente el horizonte de posibilidades de un agente dominado (B), pero no procede limitando alternativas de acción, sino abriéndolas. Lo que intento destacar es que no sólo ocultar, sino que también desocultar o abrir nuevas posibilidades de acción es una forma de influir intencionalmente en la conducta de los agentes: a veces a ellos les gustaría realizar acciones, pero carecen de oportunidades para lograrlas. 
Considerando las dos maneras de ejercer el poder entre personas e instituciones descritas por Searle, quisiera destacar algunas consecuencias de esta postura en la comprensión de la libertad de los agentes dominados. En primer lugar, en esta perspectiva dichos agentes mantendrían su voluntad: siempre poseerían un espacio de selectividad, siendo, antes que obligados, incitados a querer realizar algunas acciones. En segundo lugar, esta teoría no tematiza el eventual cambio en la cantidad de alternativas de acción que tienen los agentes sometidos al poder: el espacio de selectividad se presupone para ellos constante. Esta omisión teórica tiene como consecuencia la imposibilidad de observar dos fenómenos que permiten entender de mejor manera el ejercicio del poder: a) que dos ejercicios de poder no son equivalentes en grado, si se realizan sobre personas con cantidad de opciones dispares; y b) que no sólo ocultar alternativas, sino que también ampliarlas o desocultarlas podría ser un ejercicio de influencia intencional y, por tanto, de poder.

\section{COACCIÓN O VIOLENCIA HACIA LOS DOMINADOS}

El problema de la libertad también puede ser abordado indagando en el trato que hace Searle de un tópico transversal a las teorías del poder: la coacción u obligación por medio de la violencia física. Este concepto ha sido utilizado en las diversas propuestas teóricas principalmente con dos objetivos.

En primer lugar, la coacción se ha problematizado con la intención de distinguirla del poder. Al respecto, la posición dominante ha sido que ambos fenómenos funcionan como reversos lógicos o sustitutos. Por ejemplo, Castells $(2009,34)$ propone que, a diferencia del poder, "la pura imposición de fuerza no es una relación social, ya que lleva a la obliteración del actor social". Una idea muy similar fue desarrollada por Luhmann, quien destaca que el poder mantiene la selectividad de ambos agentes, mientras que en la coacción "las elecciones posibles de una persona que está limitada se reducen a cero" (Luhmann 2005, 14). Ambas propuestas convergen en especificar que el poder deja de existir cuando, a partir del recurso a la violencia o la fuerza física, se anula la capacidad de decisión del agente más débil. La coacción surgiría como un sustituto del poder o, más específicamente, se ejercería debido a una carencia de él (Luhmann 2005, 14). 
En segundo lugar, la coacción se ha analizado en las teorías del poder como fuente de empoderamiento. Para introducir este aspecto, simplemente podríamos replicar la pregunta efectuada por Carl Schmitt (2010, 21): “¿Por qué es que los hombres le otorgan su consentimiento al poder?”. A la cual él mismo responde (Schmitt 2010, 21):

En algunos casos, por confianza; en otros, por temor; a veces por esperanza, a veces por desesperación. En todos los casos, sin embargo, necesitan de la protección y la buscan junto al poder. Desde el punto de vista de los hombres, la relación entre protección y obediencia sigue siendo la única explicación para el poder.

Si bien la necesidad de protección representa una motivación central en la conducta humana y contribuye sin duda a explicar la dominación de hombres sobre hombres, lo cierto es que la capacidad de otorgar seguridad normalmente se ha visto garantizada por la acumulación de fuerza física — recurso aportado, en el caso del Estado moderno, típicamente por la policía y el ejército-. Aunque las principales teorías del poder han identificado dos grandes fuentes o mecanismos complementarios de empoderamiento - la capacidad de violencia física y el discurso-, entre ellas la coacción institucionalizada en el Estado sería la más fundamental, ya que permite la producción y el despliegue de significados de manera eficaz, los cuales, a su vez, aportan con la legitimación del uso estatal de la violencia. En palabras de Castells: "Los discursos disciplinarios están respaldados por el potencial uso de la violencia, y la violencia del Estado se racionaliza, interioriza y en última instancia se legitima mediante discursos que enmarcan/conforman la acción humana" (Castells 2009, 40).

Cuando Searle discute sobre la coacción, principalmente lo hace a partir de este segundo objetivo, es decir, para indagar en las distintas fuentes de poder. La fuerza física es tematizada como elemento que participa en la distinción de dos tipos de poder: poderes deónticos y de gobierno político ${ }^{7}$.

Los poderes deónticos - o relativos al deber- son aquellos en donde el poder depende típicamente de "razones para actuar", por lo

${ }^{7}$ Searle identifica un tercer tipo de poder: de trasfondo (background) o red. No obstante, para desarrollar el argumento que sigue no será necesario introducir esta tercera forma teórica. 
cual no es necesario usar la amenaza de fuerza (Searle 2010, 148). Searle utiliza como ejemplo las promesas: quien recibe una promesa comienza a tener un poder deóntico sobre quien hizo la promesa, ya que éste último tiene una "razón para actuar" de determinada manera y al margen de la amenaza de violencia. Estos poderes pueden ser negativos o positivos, según su énfasis en la constricción o habilitación del agente. Por ejemplo, una obligación representa un poder deóntico negativo, porque constriñe, mientras que una autorización sería un poder positivo, ya que habilita.

A los poderes deónticos Searle contrapone aquellos que provienen del gobierno político. Estos poderes se caracterizan por poseer un respaldo de fuerza utilizado como amenaza: los sujetos sometidos al poder siempre podrían ser arrestados, golpeados o deportados (Searle 2010, 148). Es indudable que esta distinción esconde raíces en la influyente caracterización del Estado hecha por Max Weber. Según el autor alemán, el Estado es una "comunidad humana que, dentro de un determinado territorio [...], reclama (con éxito) para sí el monopolio de la violencia física legítima" (Weber 1998, 83) ${ }^{8}$. En línea con esta tradición — que podría ser remontada al pensamiento de Hobbes-, Searle defiende que el poder del gobierno político se distingue por un monopolio de la violencia organizada y el control sobre un territorio, aunque especificando que la diferencia entre este tipo de poder y el policial o militar radica en que éste último carece por sí mismo de reconocimiento sobre su validez (Searle 2010, 163-164).

Según Searle, esta última conceptualización encierra una paradoja. El poder gubernamental se caracterizaría por descansar en un respaldo coactivo, pero más directamente por un reconocimiento o aceptación colectiva; lo paradójico es que, si bien este poder no se fundamenta en la violencia, puede continuar funcionando sólo si hay una permanente amenaza de coacción a través del ejército y la policía (Searle 2010, 163). Es indispensable, entonces, un sistema de violencia que funcione como horizonte de amenaza, a pesar de que éste no sea el fundamento distintivo del poder político, que se distancia de otros tipos de poder

${ }^{8}$ Cabe aclarar que Weber nunca planteó que la coacción física legítima sea el medio normal o único del Estado, pero sí su medio específico, ya que al resto de las asociaciones e individuos se les concede el derecho a la violencia física sólo en la medida en que el Estado lo permite (Weber 1998). 
por su reconocimiento o aceptación colectiva - proceso que algunos autores, como Weber (2008) o Habermas (1999), han denominado legitimación-.

A pesar de que Searle no sistematiza la diferencia entre coacción y poder - procedimiento que sí lleva a cabo con fenómenos como la influencia o el liderazgo-, me parece que esta distinción queda suficientemente remarcada a través de su conceptualización de la primera como recurso del segundo. La capacidad de violencia física sería un recurso necesario, aunque no suficiente, para el poder específicamente político, ya que establece un horizonte de amenaza que permite guiar la acción de los agentes (Searle 2010, 152). Mientras ella se mantenga como mecanismo de intimidación, no se elimina la voluntad de los dominados ni se produce la obliteración del agente sometido en la relación de poder. La amenaza de coacción se utilizaría, primordialmente, en la primera de las dos maneras de ejercer el poder entre personas e instituciones distinguidas por Searle; es decir, a través de ella el agente empoderado consigue que el dominado quiera hacer algo que podría no haber querido hacer de otra manera.

\section{DOS CONCEPTOS DE LIBERTAD Y LA POSTURA DE SEARLE FRENTE A LOS DOMINADOS}

Para observar de manera general el aporte de John Searle a la discusión sobre la libertad de quienes se someten al poder, ayudaría distinguir, en primer lugar, dos conceptos dominantes sobre la libertad. Siguiendo la clásica distinción delineada por Erich Fromm en 1942 y profundizada por Isaiah Berlin en $1958^{9}$, podemos identificar una versión que surge del deseo que tienen los individuos de ser dueños de sí mismos: seres pensantes, conscientes, activos y responsables de sus acciones. Ésta es la concepción positiva de la libertad, en donde las personas se identifican como "libres para" decidir una determinada forma de vida. Un ejemplo de esta perspectiva sobre la libertad se encuentra

${ }^{9}$ Comúnmente se atribuye la distinción entre libertad positiva y negativa únicamente a Isaiah Berlin. No obstante, dieciséis años antes Erich Fromm había delineado en ámbitos generales esta idea. Para conocer detalles, sugiero revisar el capítulo II de El miedo a la libertad: "La emergencia del individuo y la ambigüedad de la libertad". 
en Aristóteles $(2003,18)$, quien, en un contexto social que sostenía y validaba la esclavitud, definió al hombre libre como aquel que "es para sí mismo y no para otro". Por otro lado, existe una versión negativa de la libertad, que la entiende como el espacio en donde no hay interferencia de otro hombre o grupo de hombres en las actividades personales; ésta es la capacidad de actuar sin obstáculos, donde los agentes se sienten "libres de". Un representante de esta versión es Hobbes (1996, 145), quien entendió la libertad humana como "ausencia de oposición" o de "impedimentos externos del movimiento". Mientras la libertad positiva enfatiza la capacidad autónoma del individuo, la negativa se conforma con que haya espacios que carezcan de coacción, es decir, de intervención directa de otros seres humanos (Fromm 2008, Berlin 2005).

Desde la perspectiva de la libertad positiva - aquella comprensión de la libertad más cercana al planteamiento de Searle — ${ }^{10}$, alguien no es libre en la medida en que no es dueño de sí mismo o no puede decidir acerca de su forma de vida. Como se ha recalcado, la teoría del poder del filósofo estadounidense se caracteriza por resguardar, en todas sus formulaciones, un espacio para el querer de los agentes sometidos al poder, es decir, para la decisión autónoma de la forma de vida. Por lo tanto, si bien esta versión del poder implica una influencia intencional de los agentes empoderados, la libertad positiva de quienes se ven afectados se mantiene como supuesto basal. Esta libertad sería tan fundamental para el ejercicio del poder que, si no estuviera, éste dejaría simplemente de ejercerse, pasando a entenderse como coacción.

Al utilizar el concepto de libertad negativa, la interpretación se vuelve un poco más compleja. Desde esta perspectiva, alguien no es libre en la medida en que otros le impiden hacer lo que podría hacer de otro modo. Pues bien: ¿la teoría del poder de Searle considera obstáculos concretos para el actuar de los agentes? Aquí la respuesta es afirmativa. Como se ha señalado, una de las maneras de ejercer el poder frente

${ }^{10}$ Searle ha mostrado su postura sobre la libertad principalmente en dos de sus obras: Razones para actuar. Una teoría del libre albedrio (2000) y Libertad y neurobiología (2005). En ellas, el autor propone entender la libertad de un modo más exigente que una "carencia de impedimentos" (libertad negativa). Para él, por ejemplo, la libertad presupone racionalidad, ya que ello permite al hombre actuar con independencia de sus deseos (García 2014), es decir, con autonomía en el sentido kantiano. 
a otras personas sería impedir la observación de alternativas de acción, lo cual representa una disminución de libertad en términos negativos. Esto no significa que los agentes sometidos al poder dejen de ser libres -ya que siguen manteniendo su voluntad-, sino que en ciertos ámbitos de acción no podrían maximizar sus preferencias, ya que una parte de las alternativas les está siendo ocultada.

\section{CONCLUSIONES}

Considero que la teoría del poder realizada por Searle respalda irrestrictamente la libertad positiva de los agentes dominados, pero deja espacio para la generación de obstáculos en el actuar, es decir, para la reducción de libertad en términos negativos.

Esta garantía de libertad positiva, por un lado, y posibilidad de reducir la libertad negativamente, por el otro, nos lleva directamente a otro problema: ¿podría, en esta teoría, impedirse a tal nivel la observación de alternativas en los dominados de modo que se ejerza un poder totalizante, sin espacio para la voluntad? Aquí la respuesta sería negativa, dado que en ese nivel de reducción de alternativas para la acción no podría identificarse el fenómeno como poder, ya que si no existiera voluntad en los dominados se trataría de una imposición forzosa, es decir, de coacción. Por lo tanto, aquella forma de ejercer el poder que se caracteriza por obstaculizar alternativas en los sometidos demuestra un límite teórico: si se diera una situación extrema en que un agente tuviera sólo dos posibilidades de acción, no podría ejercerse este tipo de poder sobre él, porque si se impide la observación de una alternativa al dominado se anula por completo su voluntad, lo que implica que el fenómeno dejaría de ser identificado como poder y pasaría a entenderse como coacción. El límite para el poder, sería, entonces, la libertad de los dominados.

Esta situación impide que la teoría del poder de Searle se clasifique fácilmente entre aquellas donde el grado de poder del agente dominante se vincula negativamente con la libertad del dominado. Pero, ¿significa ello que el filósofo estadounidense se acerca a la postura contraria, es decir, entiende que el grado de poder del agente empoderado se vincula positivamente con el grado de libertad de aquel que somete? Tampoco podría sostenerse esta interpretación. Dado que Searle no desarrolla las 
herramientas teóricas para comprender el aumento de libertad entre los dominados, no considera siquiera las consecuencias de que ellos tengan una mayor libertad. Como se ha aclarado antes, el resultado de esto es la invisibilidad de algunos procesos de poder - específicamente, el ejercicio de influencia abriendo alternativas de acción, y el aumento de poder al ejercer dominio sobre un agente con mayores alternativas-. Además de esta limitación, ello determina que Searle no pueda posicionarse entre los teóricos que sostienen que el grado de poder de un agente aumenta en conjunto con la libertad de quienes domina.

Ante la pregunta principal de este ensayo: según la teoría de John Searle, "¿aumenta o disminuye el poder con un aumento de la libertad de quienes se someten a él?" sólo podría responderse lo siguiente: desde la noción de libertad positiva este concepto de poder presume como constante la voluntad de los dominados, por lo cual no podría afirmarse un aumento o disminución de la libertad; ahora bien, si se observa la libertad en términos negativos, esta teoría aceptaría que el poder puede ir en desmedro de algunos espacios de libertad de los agentes sometidos. Esto último sugiere que la teoría del poder de Searle está inclinada a aceptar la reducción de libertad de los agentes dominados, aunque no considera que ello sea la única opción posible, ya que distingue maneras de ejercer el poder que influencian a los agentes sin privarlos de voluntad ni obstaculizar sus alternativas (como los poderes deónticos). Debe resaltarse, finalmente, que el proceso de reducción de alternativas de acción —o disminución de libertad negativa - manifiesta un límite muy preciso en esta teoría: las opciones de los dominados nunca podrían eliminarse totalmente, dado que ello anularía su voluntad y transformaría el poder en coacción.

\section{REFERENCIAS BIBLIOGRÁFICAS}

Arendt, Hannah. 1970. Sobre la violencia. México: Joaquín Mortiz.

Aristóteles. 2003. Metafísica. Buenos Aires: Andrómeda.

Bauman, Zygmunt. 2007. Libertad. Buenos Aires: Losada.

Berlin, Isaiah. 2005. Dos conceptos de libertad y otros escritos. Madrid: Alianza.

Castells, Manuel. 2009. Comunicación y poder. Madrid: Alianza.

Dahl, Robert. 1957. "The Concept of Power”. Behavioral Science 2(3).

Faigenbaum, Gustavo. 2003. Conversaciones con John Searle. Buenos Aires:

Libros en Red. 
Foucault, Michel. 1999. "La ética del cuidado de sí como práctica de la libertad". Obras esenciales - Vol. III. Barcelona: Paidós.

- 2009. Vigilar y castigar. México: Siglo XXI.

Fromm, Erich. 2008. El miedo a la libertad. Barcelona: Paidós.

García, Pablo Emanuel. 2014. "La libertad en la filosofía de la mente de John Rogers Searle”. Eikasia 55.

Giddens, Anthony. 2006. La constitución de la sociedad. Buenos Aires: Amorrortu.

—. 2007. Las nuevas reglas del método sociológico. Buenos Aires: Amorrortu.

Habermas, Jürgen. 1999. Problemas de legitimación en el capitalismo tardio. Madrid: Amorrortu.

Hobbes, Thomas. 1996. Leviathan. Cambridge: Cambridge University Press.

Luhmann, Nicklas. 2005. Poder. Madrid: Anthropos.

Mann, Michael. 1986. The Sources of Social Power. Vol. 1. London: Cambridge University Press.

- 1993. The Sources of Social Power. Vol. 2. London: Cambridge University Press.

Parsons, Talcott. 1999. El sistema social. Madrid: Alianza.

Pettit, Philip. 1997. Republicanismo. Barcelona: Paidós.

Rivas, Ricardo. 2013. "Notas sobre la relación entre poder, violencia y derecho, según Arendt y Habermas”. Intersticios 38: 55-68.

Schmitt, Carl. 2010. Diálogo sobre el poder y el acceso al poderoso. Buenos Aires: Fondo de Cultura Económica de Argentina.

Searle, John. 1997. La construcción de la realidad social. Barcelona: Paidós.

— 2000. Razones para actuar. Una teoría del libre albedrío. Barcelona: Nobel.

- 2005. Libertad y neurobiología. Barcelona: Paidós

-2010. Making the Social World: The Structure of Human Civilization. London: Oxford University Press.

Walzer, Michael. 2004. Las esferas de la justicia. México: Fondo de Cultura Económica.

Weber, Max. 1998. El político y el científico. Madrid: Alianza.

—. 2008. Economía y sociedad. México: Fondo de Cultura Económica. EP 